\title{
The Sticking Out Mechanism and Growth of TiB Whiskers from TiBw/Ti-6Al-4V Composites during Sintering
}

\author{
Guorong Cui ${ }^{1,2, *}$, Bo Yang ${ }^{1,2}$, Jiabin Hou ${ }^{1,2,3}$, Wenzhen Chen ${ }^{1,2}$ and Wencong Zhang ${ }^{1,2}$ \\ 1 School of Materials Science and Engineering, Harbin Institute of Technology, Weihai 264209, China; \\ yangbogodhand@126.com (B.Y.); houjiabinwh@163.com (J.H.); nclwens@hit.edu.cn (W.C.); \\ zwinc@hitwh.edu.cn (W.Z.) \\ 2 Weihai Lightweight Materials and Forming Engineering Research Center, Weihai 264209, China \\ 3 Marine College, Shandong Jiaotong University, Weihai 264209, China \\ * Correspondence: cuiguorong2010@126.com; Tel.: +86-631-568-7209
}

Received: 30 September 2020; Accepted: 30 October 2020; Published: 4 November 2020

\begin{abstract}
The process of TiB whiskers (TiBw) squeezed out and the growth of sticking out TiBw were investigated in TiBw/Ti-6Al-4V composites via adding $5 \mathrm{vol} \% \mathrm{TiB}_{2}$ powders. $\mathrm{TiB}_{2}$ distributed along the surface of Ti-6Al-4V powders by low energy milling, resulted in the enrichment region of $\mathrm{B}$ and $\mathrm{Ti}$ elements. During sintering, TiBw nucleated and grew along the [010] direction with the diffusion of $B$ and Ti atoms in the enrichment region. Due to larger thermal expansion ratio of TiBw, the TiBw were squeezed out from the Ti matrix, and the sticking out TiBw grew gradually with the diffusion of $B$ and Ti atoms from the enrichment region. Because of the high interfacial strength between TiBw and Ti matrix, the matrix adhered to TiBw, and hindered the squeezing out of TiBw. Eventually, the cone TiBw stuck out the Ti matrix, and the other end of TiBw pinned in Ti matrix. Moreover, the diameter of TiBw near surface between $\mathrm{TiB}_{2}$ and Ti matrix was larger than other areas, resulting from the high concentration of $\mathrm{B}$ and Ti atoms. The solubilized B atoms near TiBw were absorbed by TiBw, which was considered as the other B source of TiBw.
\end{abstract}

Keywords: TiB whiskers; squeezed out; sticking out; enrichment region; thermal expansion ratio

\section{Introduction}

In recent years, Titanium (Ti) matrix composites (TMCs) have been widely used in aerospace, chemical industries, advanced military and so on, resulting from excellent high specific strength, elevated temperature strength and other properties [1-4]. Due to outstanding mechanical properties (especially ductility) and lower preparation cost, the discontinuously reinforced Ti matrix composites (DRTMCs) with ceramic reinforcements (such as TiB, TiC, TiN) were one of the hot issues [5-7]. Recently, those materials had been used in exhaust valves for high-temperature and high-pressure environments, and landing gears and aerospace engine components for saving weight, and other applications [6]. Compared to other reinforcements in TMCs, the in situ TiB whiskers (TiBw) were regarded as the best reinforcement with its advantages of high elastic modulus, clean bonding interface, similar thermal expansion coefficient between TiBw and Ti matrix [8,9].

Lots of studies about in situ TiBw reinforced Ti matrix were carried out. Feng et al. [8] investigated that the high-temperature tensile mechanical properties and fracture mechanism of in situ synthesized TiBw/TA15 matrix composites fabricated by low energy milling, pre-sintering and canned extrusion via adding $5 \%$ vol $\% \mathrm{TiB}_{2}$. Compared to TA15 alloy, the high-temperature tensile strength of composites improved significantly with the opposite plasticity. The high-temperature fracture mechanism transformed from ductile failure to the mixture of brittle fracture and ductile failure. 
Moreover, Chen et al. [9] deeply reported the influence of TiBw volume fraction on microstructure and high-temperatures mechanical properties of in situ TiBw/Ti-6Al-4V fabricated by the above method via adding $1.3 \mathrm{vol} \%, 2.6 \mathrm{vol} \%$, and $3.9 \mathrm{vol} \% \mathrm{TiB}_{2}$. Simultaneously, strengthening depended linearly on volume fractions of TiBw with the premise of good interfacial bonding between TiBw and $\mathrm{Ti}$ matrix. Additionally, Hou et al. [10] studied the grain refinement of in situ synthesized TiBw in the TiBw/Ti-15Mo-3Al-2.7Nb-0.2Si composites by low energy milling, pre-sintering and canned hot extrusion. Feng et al. [8], Chen et al. [9] and Hou et al. [10] obtained expected results in above studies; however, the process and growth of TiBw during sintering were not investigated. Harshpreet Singh [11] studied that $\mathrm{TiB}$ grows preferentially via the one-way dispersion of $\mathrm{B}$ atoms mostly along the [010] direction because of the steadiness of the B-B bonds in the [010] direction. Therefore, the faster diffusion rate in the longitudinal direction compared to the transverse direction seems to lead to the formation of needle-shaped whiskers. Tian et al. [12] studied the growth mechanism of the tubular in situ TiB crystals by coatings laser-borided. The TiB nucleates on the $\beta$-Ti with the dissolvion of $B$ atoms. Additionally, then TiBw grew along the B-B zig-zag, due to the bond of B-B being stronger than those of Ti-B and Ti-Ti. Hence, Harshpreet Singh [11] and Y.S. Tian et al. [12] did not find the process of TiBw squeezed out in their papers.

During sintering, the squeezing out process and growth of TiBw was not clarified clearly in the above studies, particularly the process of sintering. The sticking out mechanism of in situ TiBw in TMCs is worthy of exploring during sintering. Above all, the Ti- $6 \mathrm{Al}-4 \mathrm{~V}$ powders and $\mathrm{TiB}_{2}$ powders were milled by low energy milling via adding $5 \mathrm{vol} \% \mathrm{TiB}_{2}$ powders, and mixed powders were sintered.

\section{Materials and Methods}

From the process flowchart in Figure 1a, the $95 \mathrm{vol} \%$ spherical Ti-6Al-4V powders (Figure $1 \mathrm{~b}$ ) and $5 \mathrm{vol} \%$ prismatic $\mathrm{TiB}_{2}$ powders (Figure 1c) were milled by the low energy milling (LEM) (planetary ball mill), and the mixed materials were used to manufacture TiBw/Ti-6Al-4V composites. The Ti-6Al-4V powders were supplied by Shanxi Yuguang Phelly Metal Materials Co. Ltd., Shanxi, China, and the prismatic TiB2 powders were produced by Ningxia Machinery Research Institute Co. Ltd., Ningxia, China. The speed of the ball mill was $100 \mathrm{rpm}$ under the protective atmosphere of argon, and its period was $6 \mathrm{~h}$, and its weight ratio between balls and powder mixture was 5:1 [13]. After milling, the prismatic $\mathrm{TiB}_{2}$ powders were distributed along the surface of Ti-6Al-4V powders, as in Figure $1 \mathrm{~d}$. Then, the mixed powders were sealed into a silica tube under argon of $0.08 \mathrm{MPa}$. Furthermore, the silica tube was placed in a high-temperature box furnace $\left(\mathrm{SX}_{2}-20-16\right.$, Jinzhou, Liaoning, China) and sintered at $1150^{\circ} \mathrm{C}$ for $2 \mathrm{~h}$. After sintering, the silica tube was removed to the atmosphere, and cooled to room temperature. Ultimately, the desired composites were obtained, as in Figure $1 \mathrm{e}$.

(a)
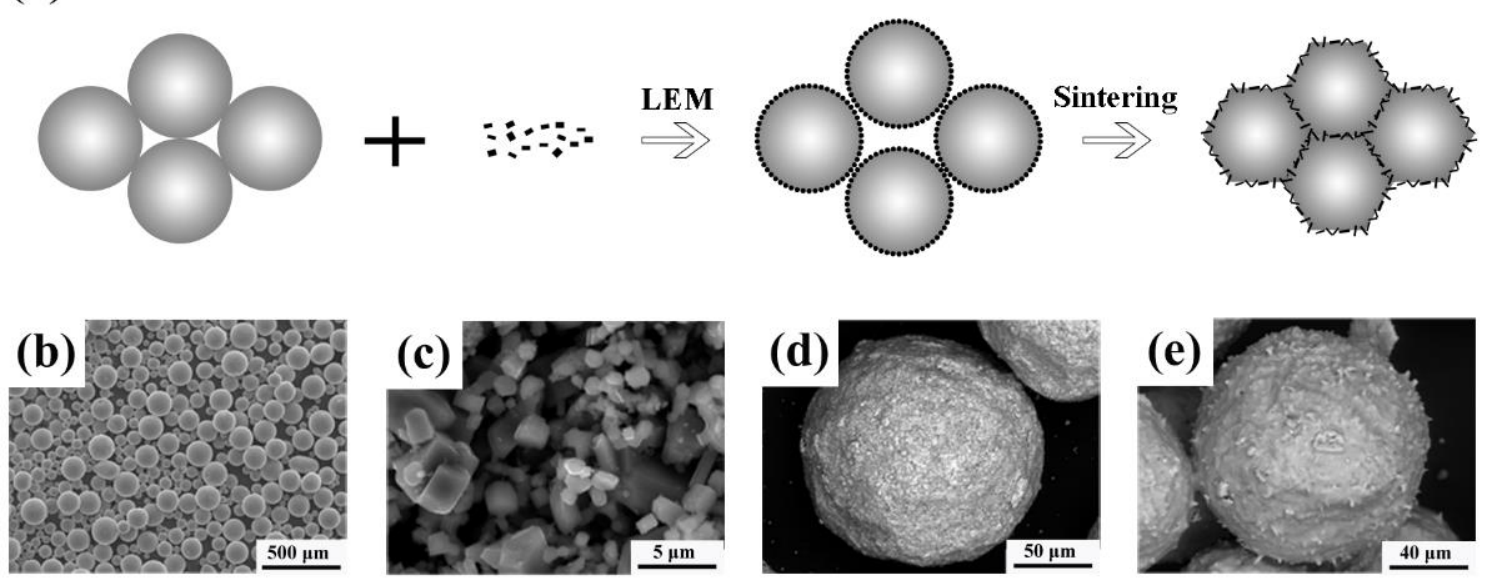

Figure 1. The process flowchart (a), SEM of spherical Ti-6Al-4V-powders (b), SEM of prismatic $\mathrm{TiB}_{2}$ powders (c), SEM of mixed powders (d), SEM of as-sintered composites (e). 
The X-ray diffraction (XRD) (Philips X' pert, Poyal philips, Amsterdam, the Netherlands) was employed to identify the phases of mixed powders and as-sintered composites. The microstructure and elemental distribution of TMCs were detected by scanning electron microscopy (SEM) with energy disperse spectroscopy (EDS) (Zeiss-MERLIN, Zürich, Switzerland). To observe and analyze the growth process of TiBw at the surface of composites during sintering, confocal scanning laser microscopy (CSLM) (Lasertec-VL2000DX, Lasertec Corporation, Tokyo, Japan) was employed to observe and collect the data with the scanning speed of 120 frames per second. The CSLM had a variable-temperature and control system to heat samples rapidly from the room temperature to $1600{ }^{\circ} \mathrm{C}$ under vacuum.

\section{Results and Discussion}

To confirm the phases precisely, the XRD of mixed powders (Figure 1d) and as-sintered composites (Figure 1e) are illustrated in Figure 2. From the XRD curve of mixed powders, $\mathrm{TiB}_{2}$ and $\mathrm{Ti}$ were detected, this result accorded closely with our analysis in Figure 1a,b. From the XRD curve of as-sintered composites, $\mathrm{TiB}$ and $\mathrm{Ti}$ phases were detected, and no $\mathrm{TiB}_{2}$ was detected. In comparison, the new peaks of the $\mathrm{TiB}$ phase appeared, along with the disappeared peaks of the $\mathrm{TiB}_{2}$ phase [14]. Meanwhile, Ma et al. [15] reported that $\mathrm{Ti}$ atoms could react with $\mathrm{TiB}_{2}$ to form $\mathrm{TiB}$ with the equation of reaction, shown as follows [16]. According to the analysis above, the chemical reaction between most $\mathrm{TiB}_{2}$ and Ti happened to form TiB during sintering.

$$
\mathrm{Ti}+\mathrm{TiB}_{2}=2 \mathrm{TiB}
$$

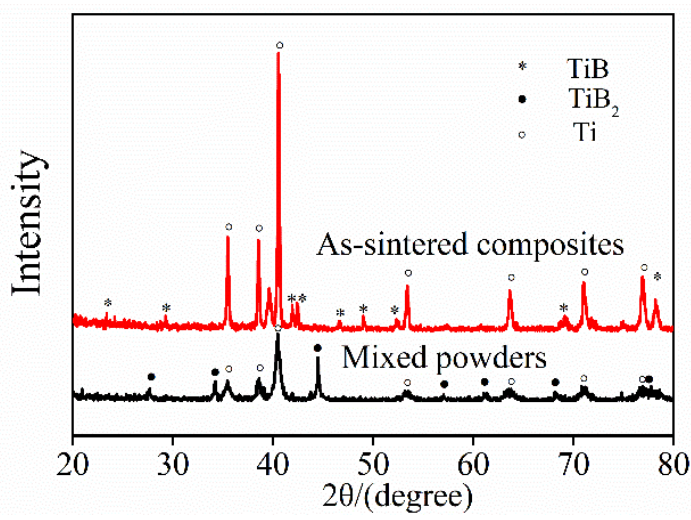

Figure 2. The XRD of mixed powders and as-sintered composites.

The SEM of TiBw and the corresponding EDS results are illustrated in Figure 3. From Figure 3a, the needled TiBw stuck out the surface of composites. The diameter of TiBw increased from the end of TiBw to the root. According to the line scanning results of sticking out needled TiBw in Figure 3c, B element content remained the same, except it was reduced at the root of TiBw. Instead, the Ti element content increased at the root. It was worth noting that the root of TiBw was wrapped by the Ti matrix (marked in black rectangle), which explained the change rule of B and Ti elements content.

To identify morphology features of TiBw pinned in the Ti matrix, the SEM and EDS results are shown in Figure 3b,d. The red circles in Figure $3 b$ represent the point positions of point scanning of TiBw pinned in Ti matrix. The diameter of TiBw decreased gradually from the surface to the internal part of the matrix. We noted that the diameter of TiBw increased from the sticking out end to the surface, which was in accord with the above results of sticking out TiBw. From the EDS results in Figure $3 \mathrm{~d}$, the $\mathrm{B}$ element content near TiBw was much lower than the far away areas, and the one in TiBw was highest, indicating that the B atoms near TiBw were absorbed to the body of TiBw to promote their growth. Based on the above analysis, one end of TiBw stuck out of the surface of the composites, the other end was pinned in the Ti matrix. The diameter of TiBw on the surface of composites was largest. 

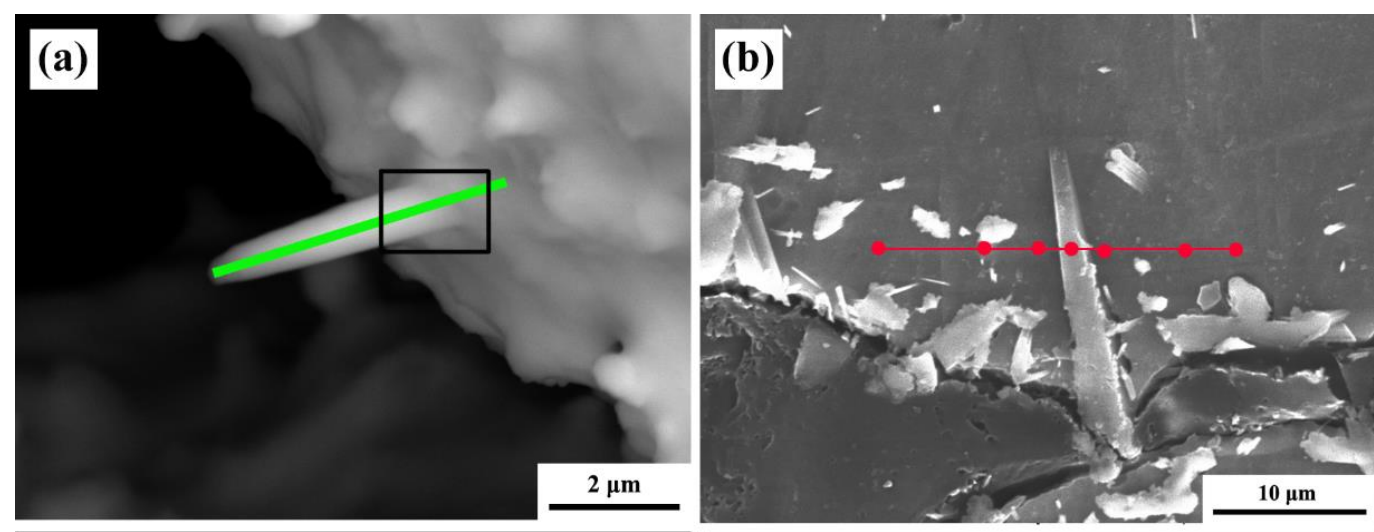

(c)

(d)
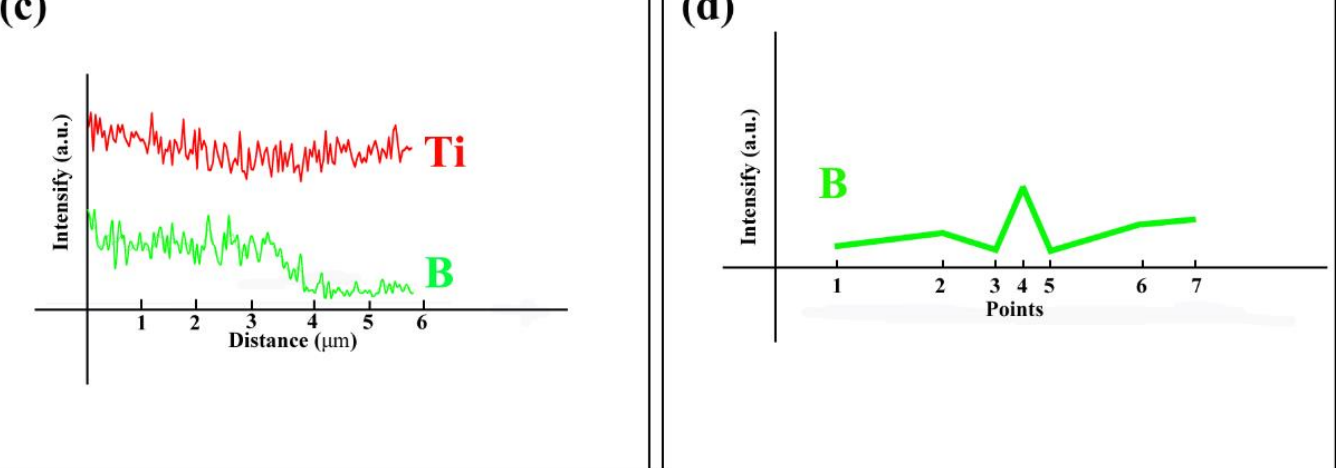

Figure 3. (a) SEM of sticking out needled TiBw, (b) SEM and of TiBw pinned in Ti matrix, (c) line scanning results of $a,(d)$ point scanning results of $b$.

To investigate the process of TiBw sticking out, the edge images of composites were observed by in-situ microscope, and the curves referred to temperature, length, diameter at the root of TiBw, volume and length/diameter along heating time are illustrated in Figure 4. According to the image in Figure $4 \mathrm{a}$ and the curve in Figure $4 \mathrm{~b}$, the needled TiBw $(\alpha)$ stuck out of the surface of TiBw/Ti-6Al-4V composites at $614 \mathrm{~s}$, with $15 \mu \mathrm{m}$ length and $1 \mu \mathrm{m}$ diameter. At $646 \mathrm{~s}$, the length of $\alpha$ increased to $20 \mu \mathrm{m}$. Ma et al. [15] investigated that a small quantity of $B$ atoms was solubilized in Ti-6Al-4V matrix, and free $\mathrm{B}$ atoms reacted with $\mathrm{Ti}$ to form needled $\mathrm{TiB}$ at the interface between $\mathrm{TiB}_{2}$ and matrix. As shown in Figure $1 \mathrm{~d}$, the $\mathrm{TiB}_{2}$ distributed along the surface of Ti- $6 \mathrm{Al}-4 \mathrm{~V}$ powders. The interface between the $\mathrm{TiB}_{2}$ and Ti matrix was the enrichment region of $B$ and Ti elements. The $B$ atoms diffused to the Ti matrix, and Ti atoms diffused to $\mathrm{TiB}_{2}$ with the beginning of sintering [17]. Furthermore, the TiBw nucleated at the interface and grew mostly along the preferential direction with the diffusion of Ti and B atoms [18].

In addition, the equation of volume expansion ratio $(\beta)$ between the TiBw and Ti matrix is shown as following [19], indicating that TiBw expanded larger than the Ti matrix during sintering. As a result, the needled TiBw were squeezed out from the matrix with the growth of TiBw along preferential direction. This is a strong evidence that the length of $\alpha$ increased quickly with the slowly increased diameter from $614 \mathrm{~s}$ to $646 \mathrm{~s}$.

$$
\beta=\frac{V_{T i B}-V_{T i}}{V_{T i}}=\frac{14.040-10.614}{10.614} \approx 23 \%
$$

At $1150{ }^{\circ} \mathrm{C}$, the softened Ti matrix adhered with the squeezing out of TiBw and squeezed out, due to the high interfacial strength between TiBw and Ti [18]. Meanwhile, the softened Ti matrix hindered the squeezing out of TiBw. This could be proved by the root of TiBw wrapped by Ti matrix in Figure $3 a$. Harshpreet Singh [11] studied that TiB grows via the one-way dispersion of B atoms mostly along the [010] direction because of the steadiness of the B-B bonds in [010] direction. Therefore, the faster diffusion rate in the longitudinal direction compared to the transverse direction seems to lead to the 
formation of needle-shaped whiskers. During the growth of needle TiBw, the TiBw were squeezed out from the Ti matrix because of the volume expansion ratio between TiBw and Ti. For the growth of sticking out $\alpha$, the B and Ti were the essential elements. Hence, the Ti and B atoms needed to diffuse to the body of $\alpha$ from the enrichment region of B and Ti elements through the interface between TiBw and Ti. However, the preferential growth direction of $\alpha$ was diameter direction instead of length with the increasing of diffusion distance. In addition, the wrapped Ti matrix increased the diffusion areas of $\mathrm{B}$ and Ti elements at the root of TiBw. As a result, the length of $\alpha$ increased very slowly from 646 to $678 \mathrm{~s}$, with the diameter increased quickly to $6 \mu \mathrm{m}$. From Figure $4 \mathrm{~b}$, the length and diameter of $\alpha$ increased slowly at holding $1000 \mathrm{~s}$ with the consumption of the B element. $\alpha$ remained the same in holding time from 1160 to $2070 \mathrm{~s}$. Eventually, the shape of sticking out TiB whiskers was the cone. The diameter of TiBw near the interface was larger than other areas in Figure $3 a$.

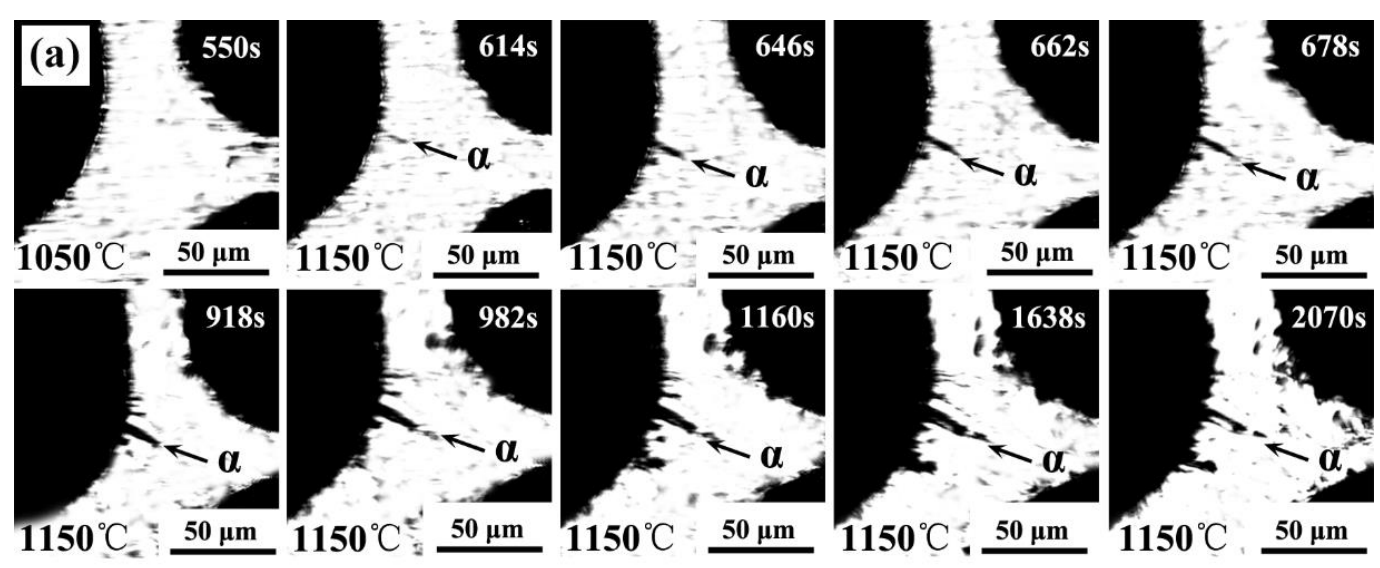

(b)

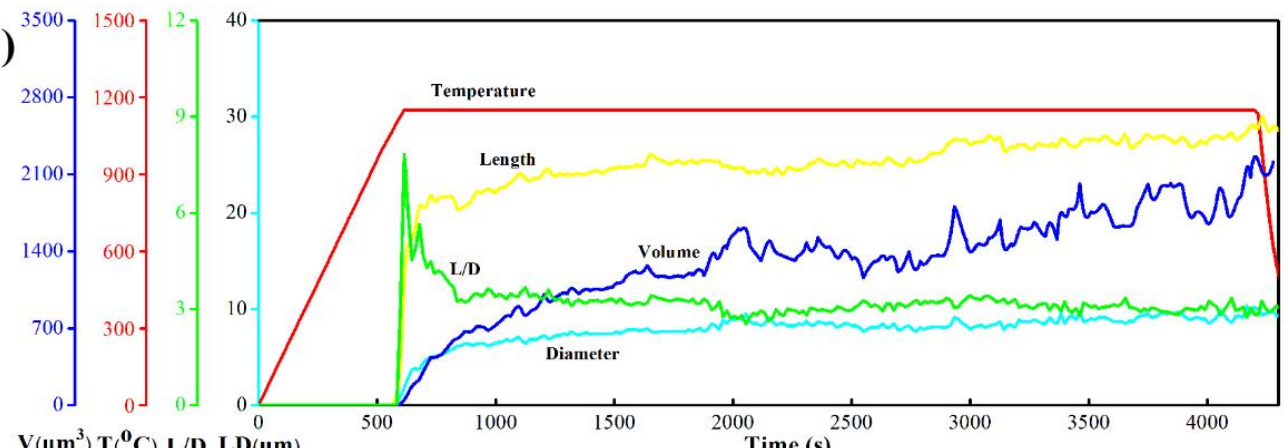

$V\left(\mu \mathrm{m}^{3}\right) \mathbf{T}\left({ }^{\mathbf{0}} \mathrm{C}\right) \mathbf{L} / \mathbf{D} \mathbf{L D}(\mu \mathrm{m})$

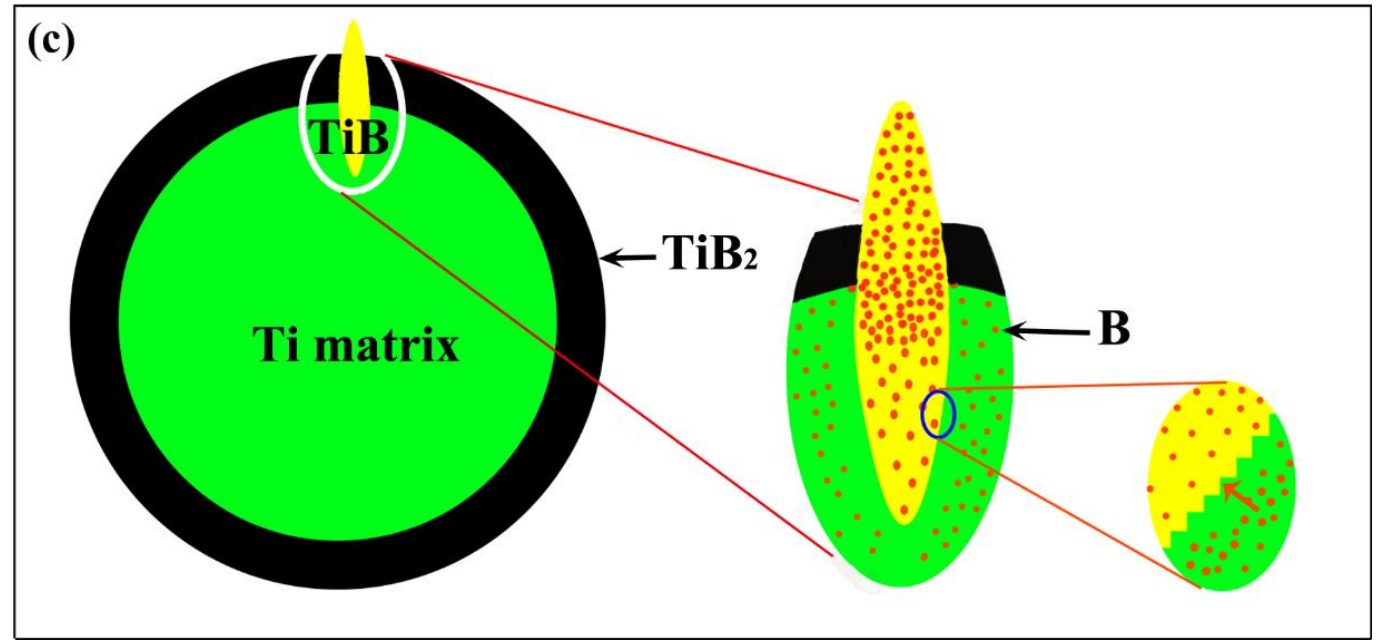

Figure 4. (a) The edge image of composites observed by in-situ microscope, (b) the curves referred to temperature and length and diameter and volume and length/diameter along heating time, (c) the schematic diagram of B atoms in the TiBw and matrix. 
According to the SEM and EDS results of the B element in Figure 3, the schematic diagram of B atoms in the TiBw and matrix was illustrated in Figure 4c. For the growth of TiBw pinned in the Ti matrix, B was the essential element. Based on the above analysis, B atoms diffused though the interface between TiBw and Ti to the body of TiBw. As we all know, the B element distributed not only in the interface of the $\mathrm{TiB}_{2}$ and Ti matrix but also in the Ti matrix. The B element content in the interface was much higher than the one in the Ti matrix, resulting that the $B$ atoms in the interface were the main source of B element. Furthermore, the solubilized B atoms near TiBw were also absorbed by TiBw through the interface, which promoted the growth of TiBw. As a result, the B element content near TiBw was lowest. According to the analysis about the growth of $\alpha$, the shape of TiBw in the Ti matrix was needle-like, with the larger diameter near the surface.

\section{Conclusions}

In this paper, the sticking out mechanism and growth of TiB whiskers (TiBw) in TiBw/Ti-6Al-4V composites during sintering were studied. The conclusions are shown as follows.

(1) During sintering, the TiBw grew along the preferential [010] direction with the diffusion of $B$ and Ti atoms. The TiBw was squeezed out from the Ti matrix, due to its larger thermal expansion ratio. The TiBw stuck quickly out the surface of as-sintered composites. The B and Ti atoms diffused from the root of sticking out TiBw to their bodies, the diffusion distance influenced the preferential direction. The shape of sticking out TiBw was the cone.

(2) The interface between $\mathrm{TiB}_{2}$ and $\mathrm{Ti}$ was the enrichment region of $\mathrm{B}$ and Ti elements, the $\mathrm{B}$ atoms mainly diffused to TiBw, and free $\mathrm{B}$ atoms were solubilized in the Ti matrix. The diameters of TiBw near the interface were larger than other areas, resulting from the high concentration of $\mathrm{B}$ and Ti elements. In addition, the solubilized $B$ atoms near the TiBw were absorbed to promote the growth of TiBw. For the TiBw in the matrix, the solubilized B atoms were also one source of the B element.

Author Contributions: Conceptualization, G.C., B.Y. and J.H.; methodology, B.Y.; W.C. and W.Z.; software, G.C.; validation, G.C., W.Z. and J.H.; investigation, W.Z.; resources, G.C.; data curation, B.Y. and J.H.; writing-original draft preparation, G.C., B.Y. and H.J.; writing-review and editing, G.C., W.Z. and W.C.; project administration, G.C.; funding acquisition, G.C. All authors have read and agreed to the published version of the manuscript.

Funding: This research was funded by the Sci-tech Major Project in Shandong Province, grant number 2018GGX102013. This research was also funded by the Natural Science Foundation of Shandong Province, grant No. ZR2019MEM037.

Conflicts of Interest: The authors declare no conflict of interest.

\section{References}

1. Attar, H.; Nisch, M.B.; Calin, M.; Zhang, L.C.; Zhuravleva, K.; Funk, A.; Scudino, S.; Yang, C.; Eckert, J. Comparative study of microstructures and mechanical properties of in situ Ti-TiB composites produced by selective laser melting, powder metallurgy, and casting technologies. J. Mater. Res. 2014, 29, 1941-1950. [CrossRef]

2. Hayat, M.D.; Singh, H.; He, Z.; Cao, P. Titanium metal matrix composites: An overview. Compos. A Appl. Sci. Manuf. 2019, 121, 418-438. [CrossRef]

3. Morsi, K.; Patel, V.V. Processing and properties of titanium-titanium boride (TiBw) matrix composites-A review. J. Mater. Sci. 2007, 42, 2037-2047. [CrossRef]

4. Tjong, S.C.; Mai, Y.W. Processing-structure-property aspects of particulate- and whisker-reinforced titanium matrix composites. Compos. Sci. Technol. 2008, 68, 583-601. [CrossRef]

5. Zheng, Y.; Williams, R.E.; Wang, D.; Shi, R.; Nag, S.; Kami, P.; Sosa, J.M.; Banerjee, R.; Wang, Y.; Fraser, H.L. Role of $\omega$ phase in the formation of extremely refined intragranular $\alpha$ precipitates in metastable $\beta$-titanium alloys. Acta Mater. 2016, 103, 850-858. [CrossRef]

6. Saito, T. A cost-effective P/M titanium matrix composite for automobile use. Adv. Perform. Mater. 1995, 2, 121-144. [CrossRef]

7. Zadra, M.; Girardini, L. High-performance, low-cost titanium metal matrix composites. Mater. Sci. Eng. A 2014, 608, 155-163. [CrossRef] 
8. Feng, Y.; Cui, G.; Zhang, W.; Chen, W.; Yu, Y. High temperature tensile fracture characteristics of the oriented TiB whisker reinforced TA15 matrix composites fabricated by pre-sintering and canned extrusion. J. Alloy. Compd. 2018, 738, 164-172. [CrossRef]

9. Chen, W.Z.; Yang, J.L.; Zhang, W.C.; Wang, M.M.; Du, D.D. Influence of TiBw volume fraction on microstructure and high-temperature properties of in situ TiBw/Ti6Al4V composites with TiBw columnar reinforced structure fabricated by pre-sintering and canned extrusion. Adv. Powder Technol. 2017, 28, 2346-2356. [CrossRef]

10. Hou, J.B.; Gao, L.; Cui, G.R.; Chen, W.Z.; Zhang, W.C.; Tian, W.G. Grain Refinement of Ti-15Mo-3Al-2.7Nb-0.2Si Alloy with the Rotation of TiB Whiskers by Powder Metallurgy and Canned Hot Extrusion. Metals 2020, 10, 126. [CrossRef]

11. Singh, H.; Hayat, M.; He, Z.; Peterson, V.K.; Das, R.; Cao, P. In situ neutron diffraction observations of Ti-TiB composites. Compos. Part A 2019, 124, 105501. [CrossRef]

12. Tian, Y.S. Growth mechanism of the tubular Tib crystals in situ formed in the coatings laser-borided on Ti-6Al-4V alloy. Mater. Lett. 2010, 64, 2483-2486. [CrossRef]

13. Hou, J.B.; Chi, F.H.; Cui, G.R.; Chen, W.Z.; Zhang, W.C. Strengthening effects of in-situ synthetic nano-TiC particles on Ti64 based nanocomposites through adding graphene nanoplatelets. Vacuum 2020, 177, 109431. [CrossRef]

14. Attar, H.; Bönisch, M.; Calin, M.; Zhang, L.-C.; Scudino, S.; Eckert, J. Selective laser melting of in situ titanium-titanium boride composites: Processing, microstructure and mechanical properties. Acta Mater. 2014, 76, 13-22. [CrossRef]

15. Ma, X.; Li, C.; Du, Z.; Zhang, W. Thermodynamic assessment of the Ti-B system. J. Alloy. Compd. 2004, 370, 149-158. [CrossRef]

16. Wang, B.; Huang, L.J.; Liu, B.X.; Geng, L.; Hu, H.T. Effects of deformation conditions on the microstructure and substructure evolution of TiBw/Ti60 composite with network structure. Mater. Sci. Eng. A 2015, 627, 316-325. [CrossRef]

17. Cherukuri, B.; Srinivasan, R.; Tamirisakandala, S.; Miracle, D.B. The influence of trace boron addition on grain growth kinetics of the beta phase in the beta titanium alloy Ti-15Mo-2.6Nb-3Al-0.2Si. Scr. Mater. 2009, 60, 496-499. [CrossRef]

18. Fan, Z.; Guo, Z.X.; Cantor, B. The kinetics and mechanism of interfacial reaction in sigma fibre-reinforced Ti MMCs. Compos. A Appl. Sci. Manuf. 1997, 28, 131-140. [CrossRef]

19. Chandran, K.S.R.; Panda, K.B.; Sahay, S.S. TiBw-reinforced Ti composites: Processing, properties, application prospects, and research needs. JOM 2004, 56, 42-48. [CrossRef]

Publisher's Note: MDPI stays neutral with regard to jurisdictional claims in published maps and institutional affiliations.

(C) 2020 by the authors. Licensee MDPI, Basel, Switzerland. This article is an open access article distributed under the terms and conditions of the Creative Commons Attribution (CC BY) license (http://creativecommons.org/licenses/by/4.0/). 